\title{
Colloque international sur la thérapie génique
}

Organisé par le Professeur Michel Boiron et le Docteur Odile CohenHaguenauer, le premier congrès international uniquement consacré aux développements récents de la thérapie génique s'est déroulé du 11 au 13 avril dernier au château de Montvillargenne, près de Chantilly (France). Il réunissait les équipes ayant le plus fortement contribué à la création des outils nécessaires à cette approche thérapeutique, celles les ayant testées sur divers modèles animaux, et celles enfin qui, aujourd'hui, pcuvent commencer d'cn faire bénéficier l'espèce humaine. Rappelons que le principe d'unc telle stratégie thérapeutique est d'apporter à l'organisme lc gène capable de produire la protéine qui lui fait défaut. Ses indications sont celles des maladies héréditaires monogéniques, c'est-à-dire causées par le dysfonctionnement d'un seul gène,

1. Briand P, Kahn A. Nouvelles orientations pour la thérapie génique. médecine/sciences 1990 ; $2: 144-9$

2. Lehn $P$. Vecteurs rétroviraux pour le tansfert de gènes dans le tissu hématopoiétique in vivo. médecine/sciences $1990 ; 6: 791-9$.

3. Rosenberg S, Aebersold P, Cornetta K, et al. Gene transfer into humans-immunotherapy of patients with advanced melanoma, using tumor-infiltrating lymphocytes modified by retroviral gene transduction. $N$ Engl $J$ Med $1990 ; 323$ : 570-8

4. Rosenfeld MA, Siegfried W, Yoshimura K, et al. In vivo transfer of a functionnal human $\alpha_{1}$-antitrypsin cDNA directly to the respiratory epithelium with a recombinant adenovirus vector. Science 1991 (sous presse).

5. Chasse JF, Levrero M, Kamoun P, et al. L'adénovirus, vecteur de thérapie génique? médecine/sciences $1989 ; 5: 331-7$.

6. Stratford-Perricaudet LD, Levrero $M$ Chasse JF, Perricaudet M, Briand P. Evaluation of the transfer and expression in mice of an enzyme-encoding gene using a human adenovirus vector. Hum Gen Ther 1990; 1 : 241-56.

7. Lemarchandel V, Montagutelli X. La recombinaison homologue : de nouvelles perspectives pour la transgenèse. médecine/sciences $1990 ; 6: 18-29$

8. Chisaka U, Capecchi M. Regionally restricted developmental defects resulting from targetted disruption of the mouse homeobox

mais aussi de maladies non génétiques, qu'il s'agisse de maladics virales, cardiovasculaires, neurodégénératives ou cancéreuses. C'est ce qu'évoquèrent les Professeurs Caskey (Houston, TX, USA), Roger Monier (IGR, Villejuif, France), Jean-Paul Lévy (ICGM, Paris, France) et Marc Girard (Institut Pasteur, Paris, France) dans leurs conférences introductives. Ces divers champs d'application, la mulitiplicité des contextes cellulaires dans lesquels le gène correcteur doit être apporté pour être efficace, font que les stratégies de transfert ne peuvent être univoques [1]. Il existe deux approches principalcs : celle utilisant des virus modifiés ayant perdu lcur autonomie réplicative et portant dans leur génome lc gène correcteur et celle des vésicules lipidiques, ou liposomes, contenant le gène à transférer. L'un des avantages théoriques de cette approche est la possibilité de diriger le transfert d'ADN dans un tissu particulier grâce à la fixation d'anticorps spécifiques de protéines localisécs à la surface des cellules à cibler. Ainsi administrés, par voie générale, ces liposomes modifiés n'iraient délivrer la molécule qu'ils renferment que dans les ccllules à traiter. Malheureusement, en l'état actucl des connaissances, comme lc rapportait le Dr Lee Leserman (Marseille, France), lorsqu'un liposome pénètre dans une cellule, son contenu se retrouve dans les lysosomes et y est dissocié. En outre, avant même cette pénétration dans la cellule ciblée, le liposome peut être capté par un macrophage circulant ct y être dissocié. Il faudra donc parvenir à modifier le liposome de telle sorte qu'il puisse efficacement apporter le gène sous une forme opérationnelle dans la cellule, avant de pouvoir envisager l'application d'une telle stratégie potentiellement idéalc. L'utilisation de virus recombinants en cst à un stade beaucoup plus avancé des investigations et c'est d'ailleurs par cette méthode que sont actuellement réalisés les premiers essais de thérapie génique chez l'homme. Avec ce type de vecteur, il n'est en revanche pas possible d'adresser précisément de l'ADN à un organe donné. Pour pallier cette difficulté, Olivicr Danos ct Jean-Michel Héard (Institut Pastcur, Paris, France) et Didicr Houssin (Hôpital Cochin, Paris, France) ont dérivé la circulation de foies de rats et démontré qu'il était ainsi possible d'administrer un gène étranger précisément à l'organe soumis à cette circulation extracorporelle. La méthode la plus utilisée consiste cepcndant à effectuer le transfert génique dans des ccllules prélevées d'un organisme et cultivées ex vivo, puis à les réintroduire après modification. Le couple modèle de cette approche est constitué des rétrovirus et du système hématopoiétique [2]. Les problèmes sont de deux ordres : d'une part, la production de rétrovirus recombinants, défectueux, en concentration élevée. L'état des connaissances en ce domaine fut rapporté par R. Mulligan et l'on peut considérer que les avancées sont en ce domaine suffisantes pour permettre l'utilisation de ces vecteurs. Le second problème est la purification des cellules souches hématopoïétiques, celles qui permettent la production renouvelée des différentes lignées de ccllules hématopoïétiques et celles, par conséquent, dans lesquelles doit être transféré le gène correcteur pour que la thérapie soit de longue durée. L'avancée des connaissances est en ce domaine moins importante, particulièrement dans l'espèce humaine où la cellule souche hématopoïétique reste beaucoup plus difficile à manipuler que dans l'espèce murine. L'addition de diverses interleukines (IL-3, IL-6) et du leukemic inhibiting factor (LIF) semblent cependant favoriser le maintien en culture des cellules totipotentes. Si l'impossibilité de 
corriger la cellule souche impose un caractère transitoire à la thérapie, l'utilisation de cellules différenciées mais ayant une durée de vie prolongée tels les lymphocytes $T$, rend raisonnable leur manipulation, et c'est d'ailleurs le transfert de gènes dans ce type cellulaire qui fit l'objet de la première autorisation de thérapie génique aux États-Unis. L'objectif était le traitement de formes graves, disséminées de mélanomes. Le Dr Michael Blaese (Bethesda, MD, USA), l'un des membres de l'équipe dirigée par les Professeurs French Anderson et Steven Rosenberg, directement impliquée dans cette approche thérapeutique, en a rapporté les derniers résultats. Les tumeurs ont été infiltrées avec des lymphocytes activés par l'interleukine 2 (TIL, tumor infiltrating lymphocytes), spécialisés dans l'élimination des cellules tumorales et ayant reçu un gène de sélection, le gène néomycine, permettant d'en suivre le devenir ( $m / s n^{\circ} 1$, vol. 7, p. 83). Cette première expérience devait permettre de mieux comprendre comment agissent ces cellules lymphocytaires dans la défense antitumorale, de savoir s'il est possible de les manipuler et de les réintroduire dans l'organisme sans occasionner de conséquences délétères. Ayant acquis la certitude qu'il en est bien ainsi [3], il s'agit maintenant de rendre ces lymphocytes plus opérationnels en leur ajoutant un gène codant pour une substance antitumorale, le TNF (tumor necrosis factor).

La seconde autorisation de traitement par thérapie génique concerne le déficit héréditaire en adénosine désaminase (ADA), déficit immunitaire combiné atteignant les cellules $B$ et les cellules $\mathrm{T}$. Les possibilités de traitements alternatifs sont dans ce cas, les greffes de moelle et l'administration de l'enzyme manquante, l'adénosine désaminase, sous une forme stabilisée (PEG-ADA). Bien que potentiel candidat depuis qu'est envisagé le principe d'une thérapie par le gène, l'absence de modèles animaux de cette maladie n'a pas facilité l'élaboration de la thérapie génique somatique des déficits en ADA. De plus, pour des raisons éthiques évidentes, elle ne peut être testée qu'en maintenant le traitement par le PEGADA dont l'efficacité, certes partielle, est néanmoins affirmée, ce qui rend délicate l'évaluation de l'efficacité thérapeutique propre du transfert de gène. Les données sont encore trop préliminaires pour affirmer qu'une amélioration clinique a été obtenue chez les malades recevant les deux traitements, mais les tests biologiques sont très encourageants.

Si les seuls essais de thérapie génique réalisés sur l'homme n'ont à ce jour mis en jeu que l'outil rétroviral, il faut néanmoins constater que son utilisation est limitée aux transferts de gènes dans des cellules qui se divisent. Or, de nombreuses cellules de l'organisme se divisent peu ou très lentement. C'est le cas des hépatocytes, qui doivent être atteints si l'on veut corriger nombre de maladies métaboliques, ou des cellules musculaires. En outre, certaines maladies comme les myopathies et la mucoviscidose $\left(\mathrm{m} / \mathrm{s} n^{\circ} 1\right.$, vol. 7, p. 84) nécessitent un traitement direct, in vivo, du fait du type et du nombre de cellules à atteindre. C'est pourquoi la recherche de vecteurs viraux adaptés à ces problèmes a fait l'objet de nombreuses recherches. A ce jour, les adénovirus défectueux recombinants, semblent, au vu des résultats obtenus chez l'animal, extrêmement prometteurs. L'équipe dirigée par Michel Perricaudet (Institut Gustave-Roussy, Villejuif, France) a particulièrement valorisé cet outil et démontré en collaboration avec Ron Crystal (NIH, Bethesda, USA), Andrea Pavirani (Transgène, Strasbourg, USA), JeanLouis Mandel (LGME, Strasbourg, France) et Pascale Briand (ICGM, Paris, France), l'efficacité des vecteurs adénoviraux pour le transfert de gènes dans les poumons par simple instillation [4] ou injection intraveineuse, les cellules musculaires par injection directe et le foie par injection intraveineuse. C'est d'ailleurs par cette technique qu'une thérapie génique somatique du déficit enzymatique murin en ornithine transcarbamylase, modèle d'une maladie humaine extrêmement sévère et pour lequel il n'existe pas de réelle thérapeutique fut obtenue [5, 6]. Il faut souligner que lorsqu'ils existent, les modèles animaux favorisent et la compréhension des maladies et l'élaboration de nouvelles voies thérapeutiques. La technique aujourd'hui classique de transgenèse permet de créer une partie de ces modèles, les autres, en particulier ceux correspondant à des maladies récessives, peuvent être obtenus par recombinaison homologue au locus du gène à muter [7]. L'un des pionniers de cette dernière technique, le Professeur Mario Capecchi (Howard Hughes, UT, USA), a ainsi décrit [8] les anomalies liées, chez l'animal, à l'absence d'expression du gène du développement hox 1-5 et montré qu'elles se rapprochaient beaucoup d'un déficit immunitaire connu sous le nom de syndrome de Di George $\left(\mathrm{m} / \mathrm{s} n^{\circ} 6\right.$, vol. 7 , à paraître).

Il est apparu au cours de cette conférence que s'il se peut que certaines maladies soient à court terme accessibles à un traitement par transfert de gènes, dont l'efficacité reste encore à prouver, les difficultés restent cependant considérables pour un grand nombre d'affections. Dans certaines anomalies héréditaires, notamment celles affectant les cellules du sang, il faut souligner que les greffes d'organes ont parallèlement fait de nombreux progrès. La possibilité d'utiliser du sang extrait de placentas humains comme source de cellules pouvant reconstituer la moelle osseuse a été commentée par Éliane Gluckman (Hôpital Saint-Louis, Paris, France). Les avantages principaux en sont la disponibilité en greffons puisqu'il en existe autant que de placentas disponibles, l'absence de problèmes éthiques liés au prélèvement de ce matériel habituellement détruit, et enfin la tolérance immunitaire qui pourrait être supérieure vis-à-vis de ce tissu que vis-à-vis d'une moelle osseuse adulte.

En conclusion, cette conférence international de très haut niveau scientifique, a permis de comparer les outils de thérapie génique, de mettre en 
relief l'intérêt des modèles animaux pour l'élaboration des stratégies et l'évaluation des résultats, d'analyser les premières applications chez l'homme, de souligner l'exigence d'une grande rigueur dans l'établissement des protocoles et dans l'évaluation des risques. La méthode en étant encore à ses balbutiements, les problèmes de sa mise en œuvre ne se posent pas encore. Ils seront liés, notamment, au caractère de haute technologie de cette approche thérapeutique qui tranche douloureuse- ment avec la répartition géographique de la majorité des malades relevant potentiellement d'une telle thérapie. Rappelons que la thérapie génique somatique s'oppose à la thérapie génique germinale. Nulle allusion n'a été faite à Montvillargenne, à cette perspective que la majorité des scientifiques rejette comme non éthique et qui, de plus est sans indication pertinente chez l'homme.

A.K.

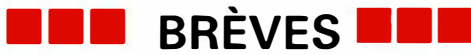

Des homéogènes chez les végétaux. Les homéogènes regroupent tous les gènes qui codent pour des protéines contenant un motif peptidique du type "boîte homéo " (homeo box). Le motif homéo a une structure de type " hélice-tour-hélice " par laquelle il interagit avec des séquences spécifiques d'ADN. Les homéogènes interviennent dans le développement (gènes homéotiques de la drosophile, gène Hox des mammifères) et dans la régulation transcriptionnelle de l'expression génétique (gènes codant pour la famille des facteurs transcriptionncls $\mathrm{Pou})\left(\mathrm{m} / \mathrm{s} n^{\circ} 7\right.$ vol. 3, p. $428 ; n^{\circ} 8$, vol. 3, p. 487 ; $n^{\circ} 3$, vol. $5, p .172$ ). Une équipe californienne vient maintenant de démontrer que les végétaux possèdent également des gènes de développement codant pour des protéines à motif homéo. Le gène knotted-1 (Kn1) est défini par des mutations dominantes qui entraînent des anomalies du développement des feuilles. L'origine de ces mutations est soit une duplication en tandem, soit l'insertion d'éléments mobiles. L'équipe californienne (Albany et Berkeley,
USA), à l'origine de cette découverte, s'est servie du motif homéo du gène $K n 1$ pour isoler d'une banque d'ADN complémentaire plusieurs clones codant pour d'autres protéines à motif homéo [1]. Les gènes correspondants semblent eux aussi ségréger avec des mutations entraînant une anomalie du développement des feuilles. Il s'agit là de la toute première démonstration que des homéogènes peuvent être impliqués dans le développement des organismes du règne végétal; les analogies de séquences entre les boîtes homéo de gènes de maïs et ceux d'homéogènes connus sont maximales avec un gène de levure (Schizosaccharomyces pombe) et de mammifères (gène humain $P r l$, pre-B cell leukemia). Cela suggère que l'origine du motif peptidique homéo remonte à un ancêtre commun des règnes végétal et animal. Tout au long de l'évolution des espèces, il semble que ce motif ait continué d'être utilisé comme un outil de la régulation de l'expression des gènes, puis de contrôle du développement. [1. Vollbrecht E, et al. Nature 1991 ; 350: 241-3.] 